\title{
Control of a Brick-Breaking Game Using Electromyogram
}

\author{
Duk Shin, Hiroyuki Kambara, Natsue Yoshimura, Yousun Kang, and Yasuharu Koike
}

\begin{abstract}
We aim to design a human-interface system to control a brick-breaking game using electromyographic (EMG) signals elicited by wrist movements. By measuring EMG signals, it is possible to predict the next stage of movement or force before motion. We filtered EMG signals through a finite impulse response filter with a cut-off frequency of $2.2 \mathrm{~Hz}$. The resulting signal is very similar to the actual tension. We calculated subtraction between flexor and extensor muscles to obtain movement of the wrist. After simple calibration, the participant was able to control a paddle to break bricks in real time using wrist movements. Consequently, we succeeded in controlling the paddle of the game. The proposed system is expected to use as a rehabilitation tool or an entertainment system.
\end{abstract}

Index Terms-EMG signals, human computer interface, rehabilitation, wrist movement.

\section{INTRODUCTION}

Muscle contraction is the fundamental unit of body motion and position control. Coordinated contractions by several muscles allow for various movements of human limbs. Human limb movements are controlled by the central nervous system (CNS). The CNS activates the muscles needed to perform a desired movement, and this activity can be observed using electromyographic (EMG) signals. It is well known EMG signal reflects the motor command from the CNS. By measuring EMG signals, it is possible to predict the next stage of movement or force before motion. Therefore, it is also possible to use EMG signals as an input device for a human interface system.

There are some approaches to using EMG signals for control of prosthetic arm or robotic hand and control command of human interfaces [1]-[5]. Saponas et. al. (2009) have used EMG signals to classify gestures on free space [6]. They demonstrated the ability to discriminate finger gestures involved finger pinching, holding a mug, and carry a bag using only EMG signals from the forearm. They used 10 EMG sensors worn in a narrow band around the upper forearm to differentiate position, pressure, tapping, and

Manuscript received May 28, 2013; revised July 15, 2013. This work was supported by JST CREST (JY210174) and JSPS KAKENHI Grant (No. 23300208). A part of this study is the result of "Brain Machine Interface Development" carried out under the Strategic Research Program for Brain Sciences by the MEXT of Japan.

D. Shin, H. Kambara, and N. Yshimura are with the Precision \& Intelligence Laboratory, Tokyo Institute of Technology, Yokohama 226-8503, Japan (e-mail: shinduk@cns.pi.titech.ac.jp; hkambara@cns.pi. titech.ac.jp; yoshimura@cns.pi.titech.ac.jp).

Y. Kang is with Department of Applied Computer Science, Tokyo Polytechnic University, Kanagawa 243-0213, Japan (e-mail: yskang@ cs.t-kougei.ac.jp).

Y. Koike is with Solution Science Research Laboratory, Tokyo Institute of Technology, Yokohama 226-8503, Japan (e-mail: koike@pi.titech.ac.jp). lifting gestures across five fingers. However, the command was corresponded to the specific gesture for controlling environments, not continuous motion in this approach.

The Bio-muse has been represented as an interface to musical synthesizers [7]. The Bio-muse translated the amplitude of EMG signals to MIDI signals directly. This has been demonstrated as an interface to music synthesizers. However, it takes time for beginners to generate the desired sound.

These two approaches are opposite extremes. Both of continuous motion and force control plays an important role in the human-computer interface (HCI) area. Therefore, the measurement of both extrinsic posture and intrinsic force are necessary for effectively interfacing a computer with a human.

Here we propose an intermediate system using EMG signals elicited by wrist motion. The proposed system enables a user to control the size (intrinsic force) and position (extrinsic posture) of a paddle and play a brick-breaking game using only wrist movement. This system can be controlled in the same way as normal input device instead of joystick.

\section{METHODS}

The brick-breaking game is composed of a laptop, an AD converter, and an EMG amp as shown in Fig. 1. To create the system, a series of preliminary experiments were first carried out to aim at data collection used for algorithm prototyping in MATLAB and DAQ-toolbox. The application fetches EMG data from the A/D-converter (NI USB-6009; National Instruments) of a $2.5 \mathrm{GHz}$ i5 processor laptop (ThinkPad; Lenovo).

The user sat on a chair in front of the desk and the laptop displayed the brick-breaking game. We performed simple calibration prior to playing because there are personal differences on EMG activities. After attaching an EMG sensor, the user was asked to shake his wrist approximately 3 S.

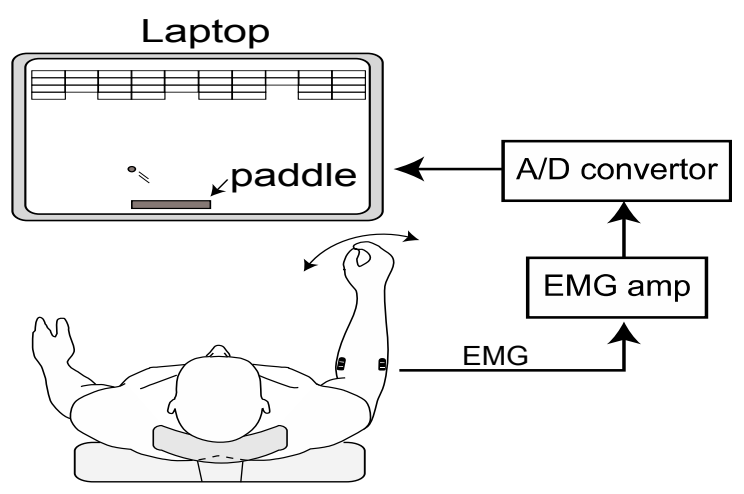

Fig. 1. Experimental setup 
We calculated the minimum and maximum of each EMG signals. Then, the position and size of the paddle was calculated from the EMG signals. After the calibration, the user could play the brick-breaking game with hand motion.

\section{A. Quasi-Tension}

Surface EMG signals are spatiotemporally convoluted action potentials of the muscle membranes, and involve not only descending central motor commands, but also reflex motor commands generated from sensory feedback signals. The muscle activation, therefore, is presumed to contribute to an increase in muscle tension and stiffness.

We recorded EMG signals using surface active electrodes in a bipolar configuration (Bagnoli ${ }^{\mathrm{TM}}$; Delsys). EMG signals were sampled at $2 \mathrm{kHz}$ with a 16-bit resolution. The signal was digitally rectified, integrated for $0.5 \mathrm{~ms}$, sampled at $200 \mathrm{~Hz}$, and passed through a second-order, low-pass filter (cut-off frequency: $2.2 \mathrm{~Hz}$ ).

Producing a signal from on referenced as $f E M G(t)$ with an impulse response of the following form [8]-[12]:

$$
f E M G(t)=\sum_{j=1}^{m} h_{j} E M G(t-j+1)
$$

where $h_{j}$ is the FIR filter, $j$ is discrete time and $E M G$ represents the rectified and integrated EMG signal. The second-order frequency response of the filter $H(s)$ is defined as

$$
H(s)=\frac{\omega_{n}^{2}}{\left(s^{2}+\zeta \omega_{n} s+\omega_{n}^{2}\right)}
$$

where $\omega_{n}$ and $\zeta$ denote natural frequency and damping coefficient, respectively.

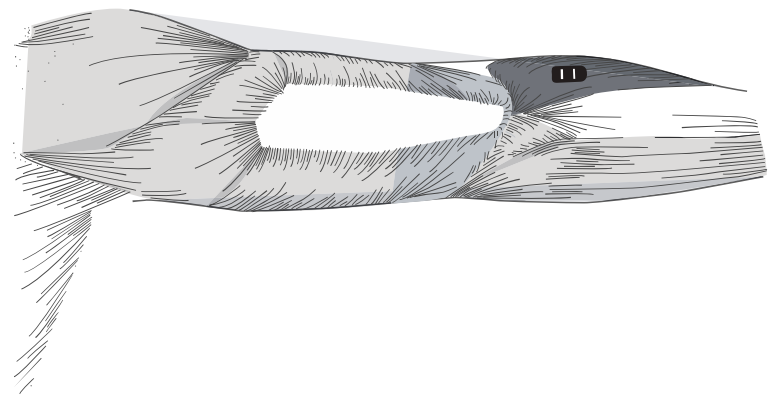

(a) Extensor carpi radialis

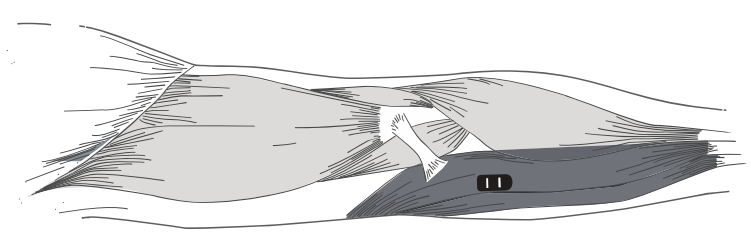

(b) Flexor carpi ulnaris

Fig. 2. The electrode positions in EMG measurement.
The impulse response of $H(s)$ is

$$
h(t)=6.44 \times\left(\exp ^{-10.80 t}-\exp ^{-16.52 t}\right)
$$

The coefficients $h_{j}$ in equation (1) were acquired by digitizing $h(t)$ [8]. Then, the filtered EMG $\left(f E M G_{i}\right)$ signals, so-called 'quasi-tension', were normalized [8].

The proposed system used 2 muscles of forearm as shown in Fig. 2. We measured EMG activities on extensor carpi radialis and flexor carpi ulnaris.

\section{B. Total Cocontraction Level}

Total cocontraction level (TCL) is calculated in order to control the size of the paddle during the experiment. TCL is defined as the summation of the absolute values of muscle tension:

$$
T C L=\sum_{i}\left|f E M G_{i}\right|
$$

TCL is proportional to joint stiffness [9]-[10].

\section{Transformation from EMG to Control Command}

The control algorithm of the paddle represented could be explained as follows.

First, a user registers the maximum and minimum values of the quasi-tension by shaking his/her wrist left side to right side. It took only 3 seconds. Next, quasi-tensions are normalized with the previously registered maximum and minimum value using equation (5).

$$
n f E M G_{i}=\frac{f E M G_{i}-f E M G_{\min }}{f E M G_{\max }-f E M G_{\min }}
$$

Then, wrist joint angle is estimated by subtracting the quasi-tension of the flexor muscle from that of the extensor muscle.

$$
\text { Angle }_{\text {wrist }}=n f E M G_{\text {extensor }}-n f E M G_{\text {flexor }}
$$

Finally, the estimated angle is changed x position required for controlling the horizontal motion of the paddle. And also the size of the paddle is determined by TCL.

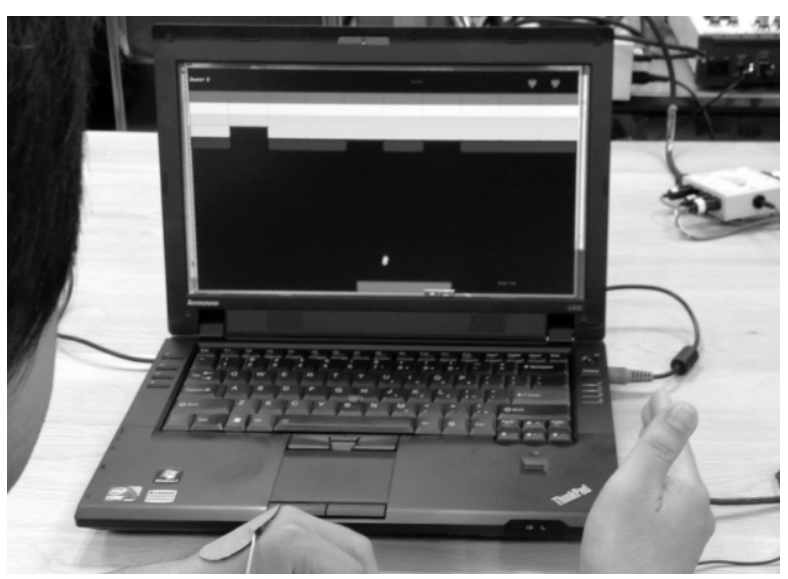

Fig. 3. The brick-breaking game using EMG signals 


\section{RESULTS}

To evaluate the effectiveness of the proposed system as a $\mathrm{HCI}$, a expert player performed two successive sessions: one for the calibration; and one for the control tasks involved to control the position and size of paddle. The calibration session was the same as described above II- $C$. After calibration, the participant was asked to play the brick-breaking game. In Fig. 3, we show the participant plays the brick-breaking game system. The player shakes his wrist on the air as if he controls a joystick, but the paddle is moved according to his wrist motion.

The EMG signals and paddle trajectory are shown in Fig. 4. These data were captured by the instruction irrelevant to playing the game. A total of 48,000 data points (24 s) of EMG and paddle data were recorded. Fig. 4 shows only $3 \sim 9$ s data because it involve well characteristic of the proposed system.

Typical plots of the EMG signals of wrist joint related muscles are shown in Fig. 4 (a) and (b). The EMG signals, which are solid thin lines, show the muscle activation during the task. Solid thick lines through the EMG signals are $f E M G$ represented 'quasi-tension'. In Fig. (c) and (d) shows the position and size of the paddle, respectively. The vertical label of (d) represents the pixel size of the paddle.

The participant could move his wrist to each of left and right target corners according to the instruction. The gray areas in the EMG signals show the cocontraction of muscle. As can be seen at EMG and $f E M G$ plots, this cocontraction and $\mathrm{S} / \mathrm{N}$ ratio of the $\mathrm{EMG}$ signals usually caused some deterioration of posture estimation. Despite that, the estimated posture (c) agrees well with the instruction. In addition, this cocontraction could succeed in control the size of the paddle. It could also provide easy to control the paddle in corner area.

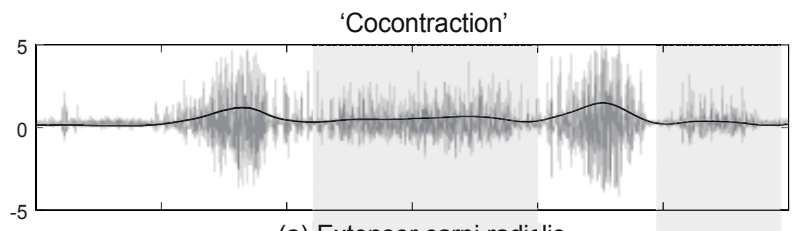

(a) Extensor carpi radialis

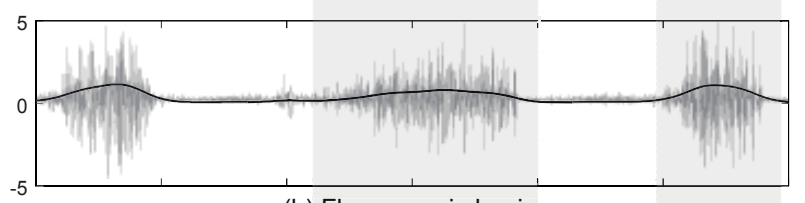

(b) Flexor carpi ulnaris

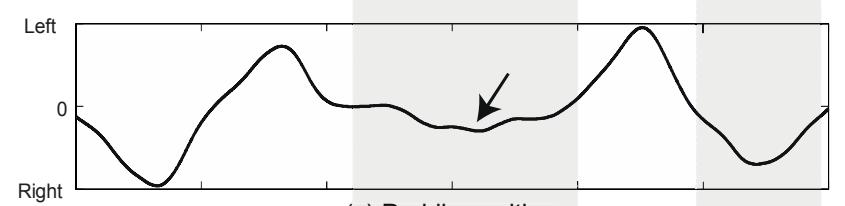

(c) Paddle position

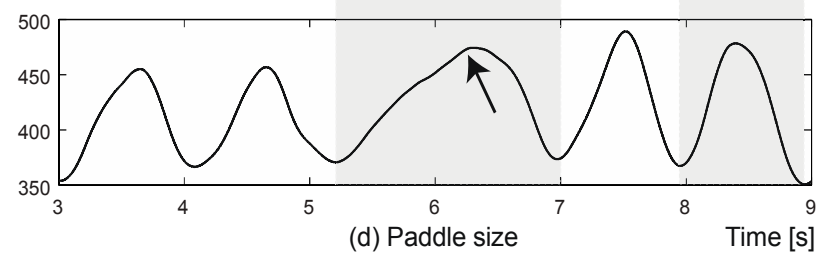

Fig. 4. The typical plot of controlling tasks.

\section{DISCUSSION}

A human interface that uses the EMG signal can be considered to have three strong advantages. First, the existing human interface could not measure the force during arm movement. In fact, it is difficult to measure the amount of force exerted by the wrist. On the contrary, the proposed method is possible to estimate the amount of exerted force because the quasi muscle tensions are obtained from EMG signals. In addition, the usage of TCL allows the estimation of joint stiffness. These points bring to large expectation to realize a humanlike movement for an active virtual human by using the internal force and stiffness of joints. Second, the EMG interface is independent of the part of the muscles; it allows that the physically disabled people could use the muscles of the shoulder or neck instead of their lost wrist muscles. Third, the EMG interface can be implemented without any delay since the EMG signal occurs before the motion. Existing human interfaces, such as a mouse and a data glove, measure a human movement after it is completed, and hence, they encounter certain difficulties in functioning in the case of some high-speed motions. On the other hand, while using the EMG interface allows to overcome the above problem.

\section{CONCLUSION}

We succeeded in controlling the position and size of the paddle by using the EMG signals the wrist muscle. This simple method provides the feasibility of the EMG interface to estimate both force and stiffness of joint. It also suggested a novel framework for an EMG-driven robot and a rehabilitation system.

\section{ACKNOWLEDGMENT}

We thank to Per-Anders Ekström for the permission of modifying his original game program.

\section{REFERENCES}

[1] D. Nishikawa, W. Yu, H. Yokoi, and Y. Kakazu, "On-line learning method for EMG prosthetic hand control," IEICE Trans. Sys. \& Info., vol. J82 D-II, no.9, pp.1510-1519, 1999

[2] K. Katsuhiro, N. Akio, O. Hiroshi et al., "The study of the system consisted of prescriptions, trainings, fabrications and maintenances," ASSISTECH Report, pp. 151-157, 2001

[3] M. Neal, "Coming to grips with artificial hand design," Design Engineering, pp.26-34, March 1993

[4] K. A. Farry, I. D .Walker, and R. G. Baraniuk, "Myoelectric teleoperation of a complex robotic hand," IEEE Trans. Robotics and Automation, vol.12, no. 5, pp.775-788, 1996

[5] O. Fukuda, J. Arita, and T. Tsuji, "An EMG-controlled omnidirectional pointing device using a HMM-based neural network," in Proc. of the IEEE International Joint Conference on Neural Networks, pp. 3195-3200, 2003

[6] T. S. Saponas, D. S. Tan, D. Morris et. al., "Enabling always-available input with muscle-computer interfaces," in Proc. of the 22nd annual ACM symposium on UIST, 2009, pp.167-176.

[7] A. Tanaka, "Musical technical issues in using interactive instrument technology with application to the BioMuse," in Proc. of ICMC, 1993, pp.124-126.

[8] Y. Koike and M. Kawato, "Estimation of dynamic joint torques and trajectory formation from surface electromyography signals using a neural network model," Biological Cyberbetics, vol. 73, pp. 291-300, 1995.

[9] D. Shin, J. Kim, and Y. Koike, "A myokinetic arm model for estimating joint torque and stiffness from EMG signals during 
maintained posture," Journal of Neurophysiology, vol. 101, pp. 387-401, 2009

[10] R. Osu, D. W. Franklin, H. Kato et.al., "Short- and long-term changes in joint co-contraction associated with motor learning as revealed from surface EMG," Journal of Neurophysiology, vol. 88, no. 2, pp. 991-1004, Aug. 2002.

[11] D. Shin, A. Katayama, K. Kim et al., "A virtual instrument system operated by electromyographic signals," Information, vol.16, no.5, pp.3275-3286, 2013.

[12] D. Shin, H. Watanabe, H. Kambara et al., "Prediction of muscle activities from electrocorticograms in primary motor cortex of primates," PLoS One, vol. 7, no. 10, p.e47992, 2012.

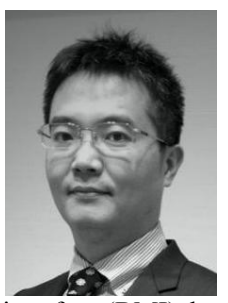

Duk Shin received the B.S. and M.S. degrees in engineering in 1996 and 1998 from Chosun University in Korea. He received Ph.D. degree in engineering in 2005 from Tokyo Institute of Technology, Tokyo, Japan.

$\mathrm{He}$ is now a project associate professor at the Precision and Intelligence Laboratory of Tokyo Institute of Technology.

His research interests include brain machine interface (BMI), brain computer interface (BCI), and bio-signal engineering. $\mathrm{He}$ is a member of Society for Neuroscience (SfN), the Japan Neuroscience Society (JNS)

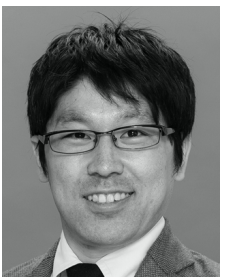

Hiroyuki Kambara received the B.Sc. degree in computer science in 2002, M.Sc. and Ph.D. degrees in engineering, in 2004 and 2007, all from Tokyo Institute of Technology, Tokyo, Japan.

$\mathrm{He}$ is now an assistant prof. at the Precision and Intelligence Laboratory of Tokyo Institute of Technology. His research interests include neural motor control and motor learning.
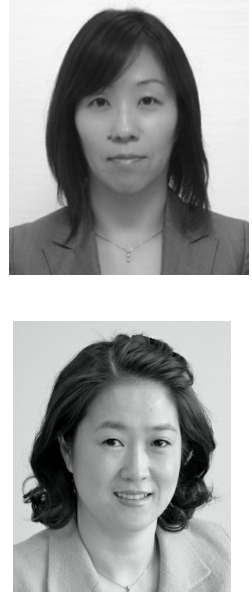

Natsue Yoshimura received her M.S degree in medical science in 2006 from Tokyo Medical and Dental University, and Ph.D. degrees in engineering in 2009 from The University of ElectroCommunications.

She is currently an assistant prof. at the Precision and Intelligence Laboratory of Tokyo Institute of Technology. Her research interests include brain machine interface.

Yousun Kang received a Ph.D. degree from Tokyo Institute of Technology in 2010. She worked with Toyota Central R\&D LABS., Inc. for three years from 2007. During 2010-2011, she was a researcher in the National Institute of Informatics, Japan.

She is currently an associate professor at Tokyo Polytechnic University. Her research interests include texture analysis, scene understanding, pattern recognition, image processing, robot vision. She is a member of the RSJ, IIEEJ and IEICE of Japan.

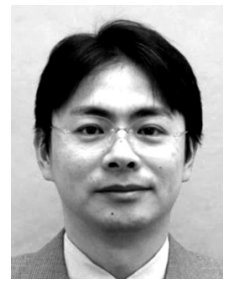

Yasuharu Koike received the B.S. and M.S. degrees in computer science in 1987, 1989 and also received Dr.Eng. degree in engineering in 1996, all from Tokyo Institute of Technology, Tokyo, Japan. He worked at Toyota Motors Corporation and Advanced Tele-communications Research (ATR) from 1989 to 1998.

$\mathrm{He}$ is currently a professor of Solution Science Research Laboratory, Tokyo Institute of Technology. His research interests include $\mathrm{BMI} / \mathrm{BCI}$ and computational motor learning. $\mathrm{He}$ is a member of Society for Neuroscience $(\mathrm{SfN})$, The Institute of Electronics, Information and Communication Engineers (IEICE), The Virtual Reality Society of Japan (VRSJ), and Japanese Neural Network Society (JNNS). 